\section{Cholinesterase Inhibitors}

Stephanie Behrens ${ }^{1}$ and JoAnn Tschanz ${ }^{1,2}$

${ }^{1}$ Department of Psychology, Utah State

University, Logan, UT, USA

${ }^{2}$ Center for Epidemiologic Studies, Utah State

University, Logan, UT, USA

\section{Synonyms}

Acetylcholinesterase inhibitors; AchEIs; CHEIs; ChEIs

\section{Definition}

A class of medications that targets the cholinergic neurotransmitter system and is used in the treatment of Alzheimer's disease and other dementias. The treatment is based on the observation of a cholinergic deficiency in the condition. Cholinesterase inhibitors (CHEI) block the activity of acetylcholinesterase, the primary enzyme that breaks down acetylcholine, allowing the neurotransmitter substance to remain in the synaptic cleft longer in order to stimulate postsynaptic receptors. The oldest CHEI, Tacrine (Cognex), is infrequently prescribed due to its unsafe side effects. Other CHEIs include Donepezil (Aricept), Rivastigmine (Exelon), and Galantamine (Razadyne). The newest addition to the CHEI class is a combination treatment, Namzaric, consisting of donepezil and memantine, the latter being an N-methyl-Daspartate-receptor antagonist. CHEIs are modestly effective in short-term (6-12 months) improvement of cognitive processes (attention, concentration, memory), functional abilities (activities of daily living), and possibly, neuropsychiatric symptoms. To date, research has shown these drugs do not show efficacy in treating mild cognitive impairment (MCI). The drugs do not prevent the further degeneration of cholinergic neurons or stop the progression of Alzheimer's disease.

\section{See Also}

Alzheimer's Dementia

\section{References and Reading}

Orgogozo, J.-M. (2003). Treatment of Alzheimer's disease with cholinesterase inhibitors. An update on currently used drugs. In K. Iqbal \& B. Winblad (Eds.), Alzheimer's disease and related disorders: Research advances (pp. 663-675). Bucharest: Ana Asian Intl. Acad. of Aging.

Rountree, S. D., Atri, A., Lopez, O. L., \& Doody, R. S. (2013). Effectiveness of antidementia drugs in delaying Alzheimer's disease progression. Alzheimer's \& Dementia, 9, 338-345.

Small, G., \& Bullock, R. (2011). Defining optimal treatment with cholinesterase inhibitors in Alzheimer's disease. Alxheimer's \& Dementia, 7, 177-184. 